\title{
On density of truth of the intuitionistic logic in one variable
}

\section{Zofia Kostrzycka}

University of Technology

Luboszycka 3, 45-036 Opole, Poland

E-mail z.kostrzycka@po.opole.pl

In this paper we focus on the intuitionistic propositional logic with one propositional variable. More precisely we consider the standard fragment $\{\rightarrow, \vee, \perp\}$ of this logic and compute the proportion of tautologies among all formulas. It turns out that this proportion is different from the analog one in the classical logic case.

Keywords: density of truth, intuitionistic logic

\section{Introduction}

Intuitionistic logic is an important subsystem of the classical one. In intuitionistic logic the effective interpretation and mechanical extraction of programs from proofs are allowed. The programming interpretation of intuitionistic propositional logic by simply typed lambda-calculus is a significant part of interest of computer scientists. The intuitionistic natural deductions are actually the same as simply $\lambda$ terms (Curry-Howard isomorphism).

For any set of formulas $A \subset F$ we define its density, denoted by $\mu(A)$, as follows:

$$
\mu(A)=\lim _{n \rightarrow \infty} \frac{|\{\alpha \in A:\|\alpha\|=n\}|}{|\{\alpha \in F:\|\alpha\|=n\}|} .
$$

The length $\|\alpha\|$ of a formula $\alpha$ is defined in the standard way and $|B|$ means the cardinality of the set $B$. If $A$ is the set of tautologies of a given logic, then $\mu(A)$ is called the density of truth of this logic. Note that $\mu(A)$ does not exists for some sets (or logics) $A$.

The density of truth, and other asymptotic properties of logics, appeared in literature. In the first place, the density was computed for various fragments of classical propositional logic (see (9), (13), (1) and (5)). The problem of equality between density of truth of classical and intuitionistic logic was raised by M. Moczurad, J. Tyszkiewicz and M. Zaionc in (11). In the present paper we take this subject up.

In (11), the densities of truth for the purely implicational fragments of classical and intuitionistic logic, in a language with one variable, were computed. The densities appeared to be the same. The authors also conjectured, that even in a language with a larger than one number of variables, the densities of truth of implicational fragments of these logics are also equal. This conjecture was refuted by Kostrzycka in (6). 
In (6), the conjecture was reformulated and it was posed that the densities of truth, for the appropriate fragments of classical and intuitionistic logics are asymptotically equal if the number of variables tends to infinity. The modified conjecture was proved by Fournier, Gardy, Genitrini and Zaionc in (4). The authors applied there a very interesting estimation for the sets of tautologies and non-tautologies of the implicational fragments of classical and intuitionistic logics.

It is possible to extend the above mentioned result to the language with implication and negation. A new problem, however, appears. The implicational-negational fragment of classical logic is functionally complete which means that any formula can be translated into an implicational-negational one. This is no longer true for the intuitionistic logic. It is known, for instance, that using $n$ variables one can define, by means of implication or negation, only finitely many formulas (up to equivalence). Whereas, even in the case of one variable, the fragment $\{\rightarrow, \vee, \neg\}$ (which is equivalent to $\{\rightarrow, \vee, \perp\}$ ) of intuitionistic logic consists of infinitely many non-equivalent formulas. They may be exposed in the so-called RiegerNishimura lattice which will be given later on. In the present paper, we consider the fragment $\{\rightarrow, \vee, \perp\}$ of intuitionistic logic, in a language with one variable $p$. The problem of computing the density of truth, for this fragment, is by no means more difficult than for any previously considered case, with finitely many non-equivalent formulas. All methods already used to compute the density, fail in the case of existing an infinite number of non-equivalent formulas. It is even not quite clear if the density ever exists. However, we give a positive answer to this question in the case of intuitionistic logic in one variable. We also provide some estimation of the density in the considered case.

\section{Semantics for intuitionistic logic}

Intuitionistic logic is a subsystem of the classical one. It can be defined by discarding, from a set of classical axioms, the law of excluded middle. Intuitionistic logic may be characterized by various types of semantics, see (10). There are considered, for instance, relational structures such as Kripke frames or Beth's tables, topological spaces or Heyting algebras. In our paper we focus on the algebraic characterization of the intuitionistic logic.

Definition 1 A Heyting algebra is a pair $(\mathcal{B}, \leq)$ where $\mathcal{B}$ is a non-empty set and $\leq$ is a lattice ordering on $\mathcal{B}$ with the least element $\perp$ and the pseudo-complement $a \rightarrow b:=\max \{c: c \wedge a \leq b\}$ for each $a, b \in \mathcal{B}$.

The greatest element is defined as $T=\perp \rightarrow \perp$ and $\neg a=a \rightarrow \perp$ is the complement of the element $a$. A valuation from the set $\operatorname{Var}$ of propositional variables to a $(\mathcal{B}, \leq)$ is a function $h: \operatorname{Var} \rightarrow \mathcal{B}$. The valuation extends to all formulas in $\{\rightarrow, \wedge, \vee, \perp\}$ as a homomorphism.

Formula $\alpha$ is valid in $(\mathcal{B}, \leq)$ if $h(\alpha)=\top$ for each valuation $h$. Formula $\alpha$ is an intuitionistic tautology if it is valid in any Heyting algebra. For example, the law of excluded middle could be falsified in the following algebra, see Figure 1.

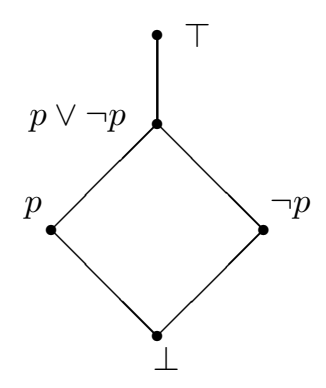


Figure 1.

\section{Generating functions}

In our paper we deal with sequences of numbers of formulas. We consider different classes of formulas (e.g. class of tautologies) and count number of formulas with the established length. To determine limits of such sequences we use generating functions. An exhaustive presentation of this method can be found, for instance, in (2) and (12). We take advantage of the Drmota-Lalley-Woods theorem; see (2), Thm. 8.13, p.71. Suppose we have two generating functions $f_{A}$ and $f_{F}$ representing, respectively, formulas from the class $A$ and all formulas. Suppose they have the same dominant singularity $\rho$ and there are suitable constants $\alpha_{1}, \alpha_{2}, \beta_{1}, \beta_{2}$ such that:

$$
f_{A}(z)=\alpha_{1}-\beta_{1} \sqrt{1-z / \rho}+O(1-z / \rho), \quad f_{F}(z)=\alpha_{2}-\beta_{2} \sqrt{1-z / \rho}+O(1-z / \rho) .
$$

Then the density of the class $A$ is given by:

$$
\mu(A)=\lim _{n \rightarrow \infty} \frac{\left[z^{n}\right] f_{A}(z)}{\left[z^{n}\right] f_{F}(z)}=\frac{\beta_{1}}{\beta_{2}} .
$$

\section{Intuitionistic logic in one variable}

In this section we consider the fragment $\{\rightarrow, \vee, \perp\}$ of intuitionistic logic, in a propositional language with one variable $p$. Let

$$
\begin{aligned}
& \alpha^{0}=\perp, \quad \alpha^{1}=p, \quad \alpha^{2}=p \rightarrow \perp, \\
& \alpha^{2 n+1}=\alpha^{2 n} \vee \alpha^{2 n-1}, \quad \alpha^{2 n+2}=\alpha^{2 n} \rightarrow \alpha^{2 n-1}
\end{aligned}
$$

In the set of all formulas $F$ we introduce an equivalence relation $\equiv$ in the standard way:

Definition $2 \varphi \equiv \psi$ if both $\varphi \rightarrow \psi$ and $\psi \rightarrow \varphi$ are intuitionistic tautologies.

Every formula from our language $F$ falls into one of the equivalence classes $A^{m}=\left[\alpha^{m}\right]_{\equiv}$. Therefore, up to this equivalence relation on the classes of formulas $A^{m}$, the quotient algebra rises to the so-called Rieger - Nishimura lattice $\mathcal{R}$, which is a single-generated free Heyting algebra (see Figure 2).

The above algebra is a nice example of a finitely generated free algebra, which is infinite. Let us stress, that $A^{\omega}$ is the class of intuitionistic tautologies in the considered language. Our purpose is to compute the density $\mu\left(I N T_{\{p\}}^{\{\rightarrow, \vee \perp\}}\right)=\mu\left(A^{\omega}\right)$ if it exists. This is directly related to computing (and proving the existence) the density of the class $A^{m}$ for each $m \geq 0$. There are infinitely many classes, so the methods which are effective in the cases of finite algebras (and logics) must be now modified.

The length of a formula is defined as follows:

Definition 3

$$
\|p\|=1, \quad\|\perp\|=1, \quad\|\phi \rightarrow \psi\|=\|\phi\|+\|\psi\|, \quad\|\phi \vee \psi\|=\|\phi\|+\|\psi\|
$$




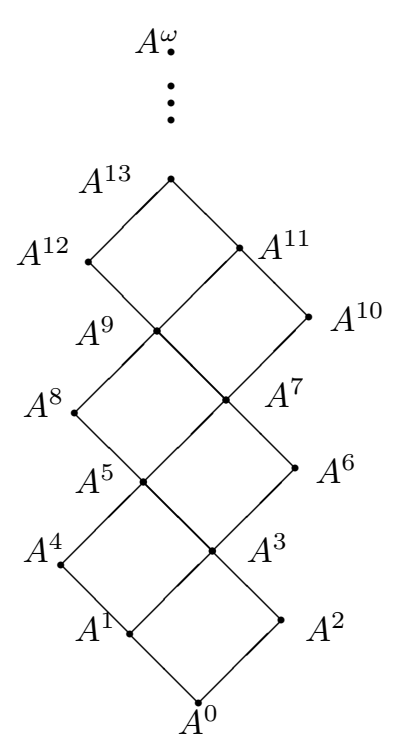

Zofia Kostrzycka

Figure 2.

By $F_{n}$ we mean the set of formulas of the length $n$ and, by $\left|F_{n}\right|$, the cardinality of $F_{n}$. We will also consider some subclasses of $F_{n}$. For any $B \subset F$, we take $B_{n}=B \cap F_{n}$ and, by $\left|B_{n}\right|$, we denote the cardinality of the class $B_{n}$.

Lemma 4 The generating function $f$ for the numbers $\left|F_{n}\right|$ is the following:

$$
f(z)=\frac{1-\sqrt{1-16 z}}{4} .
$$

Proof. The number $\left|F_{n}\right|$ of formulas from $F_{n}$ is given by the recursion:

$$
\left|F_{0}\right|=0, \quad\left|F_{1}\right|=2, \quad\left|F_{n}\right|=2 \sum_{i=1}^{n-1}\left|F_{i}\right|\left|F_{n-i}\right| .
$$

Hence the function $f$ has to fulfil the equation: $f(z)=2 f^{2}(z)+2 z$. With the boundary condition: $f(0)=0$ we obtain 4 .

\section{Quotient algebras}

In this section we give a practical approach to the problem of computing the density of truth in the case of infinite Rieger-Nishimura lattice.

It can be immediately seen from the definition that the density $\mu$ is finitely additive. So, if $A$ and $B$ are disjoined classes of formulas such that $\mu(A)$ and $\mu(B)$ exist, then $\mu(A \cup B)$ exists as well and

$$
\mu(A \cup B)=\mu(A)+\mu(B) .
$$


Unfortunately, the density $\mu$ is not countably additive. We only have the following inequality:

$$
\mu\left(\bigcup_{i=0}^{\infty} A_{i}\right) \geq \sum_{i=0}^{\infty} \mu\left(A_{i}\right)
$$

In the case of Rieger - Nishimura lattice $\mathcal{R}$, we have $\bigcup_{i=0}^{\infty} A^{i} \cup A^{\omega}=F$ and hence we get $\mu\left(\bigcup_{i=0}^{\infty} A^{i} \cup A^{\omega}\right)=1$. In this section we show that $\mu\left(A^{i}\right)$ exists for any $i \in \mathbb{N}$. In Section 9 we prove the existence of $\mu\left(A^{\omega}\right)$. Before we are able to do it, we consider finite quotient algebras obtained from the Rieger - Nishimura lattice $\mathcal{R}$.

Definition 5 Let $(\mathcal{B}, \leq)$ be a Heyting algebra. A nonempty set $\mathcal{D} \subset \mathcal{B}$ is a filter if: 1) a $\wedge b \in \mathcal{D}$, for any $a, b \in \mathcal{D} ; 2)$ if $a \in \mathcal{D}$ and $a \leq c$, then $c \in \mathcal{D}$.

By $\left[A^{2 n-1}\right)$ we mean a filter in the algebra $\mathcal{R}$ generated by the element $A^{2 n-1}$. Formally, $\left[A^{2 n-1}\right)=\left\{\alpha \in F: \alpha^{2 n-1} \rightarrow \alpha \in A^{\omega}\right\}, n \geq 1$.

We consider a sequence of quotient algebras obtained from the algebra $\mathcal{R}$ by division by the filters: $\left[A^{2 n-1}\right)$ for $n \geq 2$. Every such algebra consists of $2 n$ elements, so we will denote it as $A L_{2 n}$. We have:

$$
A L_{4}:=\mathcal{R} /\left[A^{3}\right), \quad A L_{6}:=\mathcal{R} /\left[A^{5}\right), \ldots A L_{2 n}:=\mathcal{R} /\left[A^{2 n-1}\right), \ldots
$$
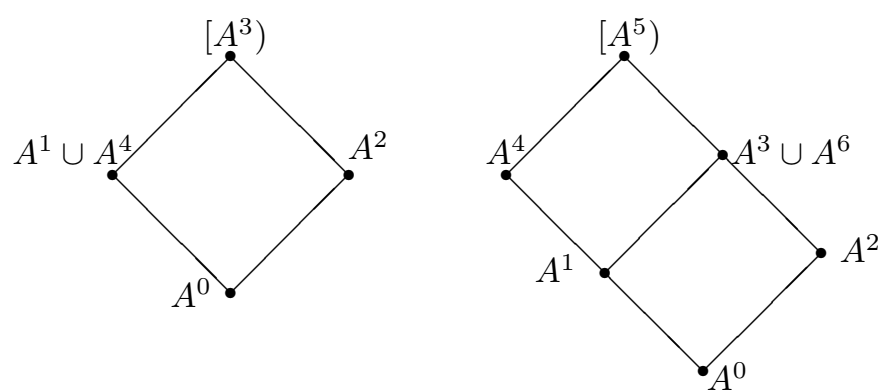

Figure 3. Diagrams of $A L_{4}$ and $A L_{6}$.

Let us notice that the filters $\left[A^{2 n-1}\right), n \geq 2$ form the following decreasing sequence:

$$
\left[A^{3}\right) \supset\left[A^{5}\right) \supset \ldots \supset\left[A^{2 n-1}\right) \supset \ldots \supset A^{\omega} .
$$

Theorem 6 The density $\mu\left(A^{k}\right)$ exists for any $k \in \mathbb{N}$.

Proof. The idea of the proof is to take some finite quotient algebra $A L_{2 n}$ such that $2 n>k-3$ and show that the appropriate system of $2 n$ equations fulfils the assumptions of the Drmota-Lalley-Woods theorem.

\begin{tabular}{|c|c|c|c|c|c|c|c|c|c|}
\hline$\rightarrow$ & $A^{0}$ & $A^{1} \cup A^{4}$ & $A^{2}$ & {$\left[A^{3}\right)$} & V & $A^{0}$ & $A^{1} \cup A^{4}$ & $A^{2}$ & {$\left[A^{3}\right)$} \\
\hline$A^{0}$ & {$\left[A^{3}\right)$} & {$\left[A^{3}\right)$} & {$\left[A^{3}\right)$} & {$\left[A^{3}\right)$} & $A^{0}$ & $A^{0}$ & $A^{1} \cup A^{4}$ & $A^{2}$ & {$\left[A^{3}\right)$} \\
\hline$A^{1} \cup A^{4}$ & $A^{2}$ & {$\left[A^{3}\right)$} & $A^{2}$ & {$\left[A^{3}\right)$} & $A^{1} \cup A^{4}$ & $A^{1} \cup A^{4}$ & $A^{1} \cup A^{4}$ & {$\left[A^{3}\right)$} & {$\left[A^{3}\right)$} \\
\hline$A^{2}$ & $A^{1} \cup A^{4}$ & $A^{1} \cup A^{4}$ & {$\left[A^{3}\right)$} & {$\left[A^{3}\right)$} & $A^{2}$ & $A^{2}$ & {$\left[A^{3}\right)$} & $A^{2}$ & {$\left[A^{3}\right)$} \\
\hline$\left[A^{3}\right)$ & $A^{0}$ & $A^{1} \cup A^{4}$ & $A^{2}$ & {$\left[A^{3}\right)$} & {$\left[A^{3}\right)$} & {$\left[A^{3}\right)$} & {$\left[A^{3}\right)$} & {$\left[A^{3}\right)$} & {$\left[A^{3}\right)$} \\
\hline
\end{tabular}
Formally, the proof should proceed by induction but we present here only the basic step. Let us consider the algebra $A L_{4}$. The operations $\{\rightarrow, \vee\}$ in the algebra are given by the following truth-tables: 
Table 1.

The appropriate generating functions $f_{0}, f_{1}+f_{4}, f_{2}, f_{[3)}$ for the classes $A^{0}, A^{1} \cup A^{4}, A^{2},\left[A^{3}\right)$ fulfil the following system of equations:

$$
\left\{\begin{array}{cl}
f_{0}(z)= & f_{[3)}(z) \cdot f_{0}(z)+\left[f_{0}(z)\right]^{2}+z \\
\left(f_{1}+f_{4}\right)(z)= & f_{[3)}(z) \cdot\left(f_{1}+f_{4}\right)(z)+f_{2}(z) \cdot\left[f_{0}(z)+\left(f_{1}+f_{4}\right)(z)\right]+2 f_{0}(z) \cdot\left(f_{1}+f_{4}\right)(z) \\
& +\left[\left(f_{1}+f_{4}\right)(z)\right]^{2}+z \\
& f_{[3)}(z) \cdot f_{2}(z)+\left(f_{1}+f_{4}\right)(z) \cdot\left[f_{0}(z)+f_{2}(z)\right]+2 f_{0}(z) \cdot f_{2}(z)+\left[f_{2}(z)\right]^{2} \\
f_{2}(z)= & f(z)-\left[f_{0}(z)+\left(f_{1}+f_{4}\right)(z)+f_{2}(z)\right]
\end{array}\right.
$$

From the Drmota-Lalley-Woods theorem we conclude that all the functions have the same dominant singularity. Actually, it is the same singularity as the one of the function $f$. Hence $z_{0}=1 / 16$ is the common singularity and the densities of the classes $A^{0}, A^{1} \cup A^{4}, A^{2},\left[A^{3}\right)$ exist.

Analogous situation holds for the algebra $A L_{2 n}$ for any $n>\mathbb{N}$. All involved functions have the same dominant singularity $z_{0}=1 / 16$. Hence densities of all classes of formulas from $A L_{2 n}$ exist. Among these classes we have the class of true formulas in $A L_{2 n}$, which is denoted as $\left[A^{2 n-1}\right)$.

From Theorem 6 and inclusions $[8]$ it follows that:

Corollary 7 The density $\mu\left(\left[A^{2 n-1}\right)\right)$ exists for any $n \in \mathbb{N}$ and the following inequalities hold:

$$
\mu\left(\left[A^{3}\right)\right) \geq \mu\left(\left[A^{5}\right)\right) \geq \ldots \geq \mu\left(\left[A^{2 n-1}\right)\right) \geq \ldots
$$

\section{Calculation of the basic generating functions}

In this section we calculate the generating functions for the classes: $A^{0}, A^{1} \cup A^{4}, A^{2},\left[A^{3}\right)$. To solve the system of equations 97 we use strictly algebraical methods, i.e. division by filters. The method is presented with all details in (7) and (8). Roughly speaking, we take two filters: $\left[A^{2}\right)$ and $\left[A^{1}\right)$, and two quotient algebras: $A L_{4} /{ }_{\left[A^{2}\right)}$ and $A L_{4} /\left[A^{1}\right)$, which are two-element algebras. In the first one we have classes: $\left[A^{2}\right)$ and $A^{0} \cup A^{1} \cup A^{4}$, in the second one: $\left[A^{1}\right)$ and $A^{0} \cup A^{2}$.

Let us consider the second algebra $A L_{4} /_{\left[A^{1}\right)}$ and let $f_{0}^{*}:=f_{0}+f_{2}$. We can draw an appropriate truth table for the operations: $\rightarrow, \vee$ and obtain a suitable equation for the function $f_{0}^{*}$. It is:

$f_{0}^{*}=f_{[1)} \cdot f_{0}^{*}+\left(f_{0}^{*}\right)^{2}+z$. Also: $f_{[1)}=f-f_{0}^{*}$. Hence after simplification we get: $f_{0}^{*}=\frac{z}{1-f}$.

Analogously, we obtain the generating function $f_{0}^{* *}$ for the class $A^{0} \cup A^{1} \cup A^{4}$ in the algebra $A L_{4} /\left[A^{2}\right.$ ). It is: $f_{0}^{* *}=\frac{2 z}{1-f}$. Hence $f_{0}^{* *}=2 f_{0}^{*}$. Having the functions $f_{0}^{*}$ and $f_{0}^{* *}$ we can determine the generating functions for the classes from algebra $A L_{4}$.

Lemma 8 The generating function $f_{0}$ for the class $A^{0}$ is the following:

$$
f_{0}(z)=\frac{1}{4}\left(1+3 f^{*}-f-\sqrt{\left(1+3 f^{*}-f\right)^{2}-8 z}\right),
$$

where $f$ is defined by (4) and $f^{*}=\frac{z}{1-f}$.

Proof. In the first equation from (9) we put: $f_{[3)}=f-f_{0}^{*}-f_{0}^{* *}+f_{0}$ and $f_{0}^{* *}=2 f_{0}^{*}$. Then we obtain an equation with the one unknown function $f_{0}$.

$$
f_{0}(z)=\left[f(z)-3 f_{0}^{*}(z)+f_{0}(z)\right] \cdot f_{0}(z)+\left[f_{0}(z)\right]^{2}+z .
$$


It is a quadratic equation and after solving it with the condition $f_{0}(0)=0$ we obtain 11 .

Having the function $f_{0}$ we obtain the other generating functions from $A L_{4}$.

$$
\left(f_{1}+f_{4}\right)=2 f^{*}-f_{0}, \quad f_{2}=f^{*}-f_{0}, \quad f_{[3)}=f-f_{0}-\left(f_{1}+f_{4}\right)-f_{2} .
$$

We expand the functions $f, f_{0},\left(f_{1}+f_{4}\right), f_{2}$ and $f_{[3)}$ in a neighborhood of $z_{0}=1 / 16$ and, by use of the transfer lemma, we obtain:

Lemma 9 The densities of the classes of formulas from the algebra $A L_{4}$ are the following:

$$
\mu\left(A^{0}\right) \approx 0.069, \quad \mu\left(A^{1} \cup A^{4}\right) \approx 0.153, \quad \mu\left(A^{2}\right) \approx 0.042, \quad \mu\left(\left[A^{3}\right)\right) \approx 0.736
$$

Analogously, we determine the generating functions for the classes from algebra $A L_{6}$. Obviously, the functions $f_{0}$ and $f_{2}$ are already given. Using again division by filters, we are able to solve the appropriate system of six equations. It is not difficult to define the functions $f_{1}, f_{4},\left(f_{3}+f_{6}\right), f_{[5)}$ explicitly, expand them around $z_{0}=1 / 16$, use the transfer lemma and obtain:

Lemma 10 The densities of the classes from the algebra $A L_{6}$ are the following:

$$
\mu\left(A^{1}\right) \approx 0.1315, \quad \mu\left(A^{3} \cup A^{6}\right) \approx 0.0244, \quad \mu\left(A^{4}\right) \approx 0.02164, \quad \mu\left(\left[A^{5}\right)\right) \approx 0.71099
$$

\section{Upper bound of the density of INT}

In this section we estimate the density of $I N T_{\{p\}}^{\{\rightarrow, \vee, \perp\}}$ by densities of the filters $\left[A^{2 k-1}\right)$. Let us consider the algebra $A L_{2 k}$; see Figure 4.
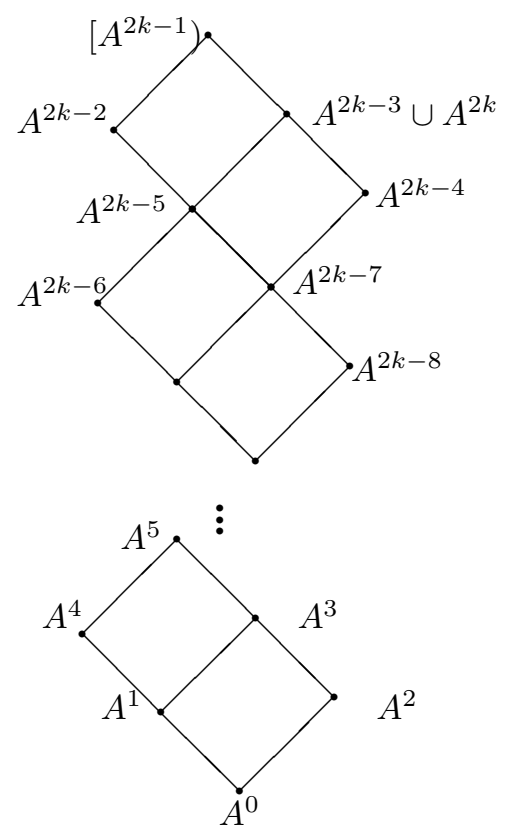

Figure 4. Diagram of $A L_{2 k}$. 
Directly from the diagram of $A L_{2 k}$ we can read out how formulas from the class $\left[A^{2 k-1}\right)$ are obtained. They arise as implications from $A^{0}$ to any other class, and from $A^{1}$ to any other class, with the exception of $A^{0}$ and $A^{2}$, and so on. They also arise as disjunctions between formulas from $A^{2 k-4} \cup A^{2 k-3} \cup A^{2 k}$ and $A^{2 k-2}$, and from $\left[A^{2 k-1}\right)$ and any other class. To write it precisely, we introduce the following abbreviation: $\{A \odot B\}=\left\{\alpha \in F: \exists_{\alpha_{1} \in A} \exists_{\alpha_{2} \in B} \quad \alpha=\alpha_{1} \odot \alpha_{2}\right\}$ for $\odot \in\{\rightarrow, \vee\}$. Hence we have:

$$
\begin{aligned}
& {\left[A^{2 k-1}\right)=\left\{A^{0} \rightarrow F\right\} \cup\left\{A^{1} \rightarrow\left(F \backslash\left(A^{0} \cup A^{2}\right)\right)\right\} \cup\left\{A^{2} \rightarrow\left(F \backslash\left(A^{0} \cup A^{1} \cup A^{4}\right)\right)\right\} \cup \ldots} \\
& \ldots \cup\left\{\left(A^{2 k-3} \cup A^{2 k}\right) \rightarrow\left(A^{2 k-3} \cup A^{2 k} \cup\left[A^{2 k-1}\right)\right)\right\} \cup\left\{A^{2 k-2} \rightarrow\left(A^{2 k-2} \cup\left[A^{2 k-1}\right)\right)\right\} \cup \\
& \cup\left\{A^{2 k-2} \vee\left(A^{2 k-4} \cup A^{2 k-3} \cup A^{2 k}\right)\right\} \cup\left\{\left(A^{2 k-4} \cup A^{2 k-3} \cup A^{2 k}\right) \vee A^{2 k-2}\right\} \cup \\
& \cup\left\{\left[A^{2 k-1}\right) \rightarrow\left[A^{2 k-1}\right)\right\} \cup\left\{\left[A^{2 k-1}\right) \vee\left[A^{2 k-1}\right)\right\} \cup\left\{\left[A^{2 k-1}\right) \vee\left(F \backslash\left[A^{2 k-1}\right)\right)\right\} \cup \\
& \cup\left\{\left(F \backslash\left[A^{2 k-1}\right)\right) \vee\left[A^{2 k-1}\right)\right\} .
\end{aligned}
$$

Of course, the sum written above as '...' is finite in the case of finite algebra $A L_{2 k}$. After simplification, the above formula may by transformed into the following one:

$$
\begin{aligned}
f_{[2 k-1)}= & {\left[f_{0} \cdot f+f_{1} \cdot\left(f-f_{0}-f_{2}\right)+f_{2} \cdot\left(f-f_{0}-f_{1}-f_{4}\right)+f_{3} \cdot\left(f-f_{0}-f_{1}-f_{2}-f_{4}\right)+\ldots\right.} \\
& \ldots+f_{2 k-2} \cdot\left(f-f_{0}-f_{1}-\ldots-f_{2 k-3}-f_{2 k}\right)+ \\
& +\left(f_{2 k-3}+f_{2 k}\right) \cdot\left(f-f_{0}-f_{1}-\ldots-f_{2 k-4}-f_{2 k-2}\right)+ \\
& \left.+2 f_{2 k-2} \cdot\left(f_{2 k-4}+f_{2 k-3}+f_{2 k}\right)\right] /(1-2 f)
\end{aligned}
$$

Note that all generating functions, related to the algebra $A L_{2 n}$, are involved in the above equation. It is possible to determine the functions $f_{[2 n-1)}$ explicitly, for any $n \in \mathbb{N}$. We skip all details, the same methods as in (7) and (8) should be used here. Let us add that the growing index $n$ involves increase of the number of nested square roots in the formula defining $f_{[2 n-1)}$. It is not difficult to compute the density of the class of valid formulas for the next two algebras: $A L_{8}$ and $A L_{10}$. They are as follows: $\mu\left(\left[A^{7}\right)\right) \approx 0.709016$ and $\mu\left(\left[A^{9}\right)\right) \approx 0.709011$. From the inequality 10$]$ we get:

Observation 11 If the density of $\mu\left(A^{\omega}\right)$ exists then $\mu\left(A^{\omega}\right)<0.709011$.

\section{Lower bound of the density of INT}

Let us consider the algebra $\mathcal{R}$. As we see in Figure 1, the formulas from the class $A^{\omega}$ are either implications or some special disjunctions. In these disjunctions at least one formula comes from $A^{\omega}$. Hence, analogously as in the previous section, we may write down the set of intuitionistic tautologies $A^{\omega}$ as follows:

$$
\begin{aligned}
& A^{\omega}=\left\{A^{0} \rightarrow F\right\} \cup\left\{A^{1} \rightarrow\left(F \backslash\left(A^{0} \cup A^{2}\right)\right)\right\} \cup\left\{A^{2} \rightarrow\left(F \backslash\left(A^{0} \cup A^{1} \cup A^{4}\right)\right)\right\} \cup \\
& \cup\left\{A^{3} \rightarrow\left(F \backslash\left(A^{0} \cup A^{1} \cup A^{2} \cup A^{4}\right)\right)\right\} \cup\left\{A^{4} \rightarrow\left(F \backslash\left(A^{0} \cup A^{1} \cup A^{2} \cup A^{3} \cup A^{6}\right)\right)\right\} \cup \\
& \cup\left\{A^{5} \rightarrow\left(F \backslash\left(A^{0} \cup A^{1} \cup A^{2} \cup A^{3} \cup A^{4} \cup A^{6}\right)\right)\right\} \cup \\
& \cup \ldots . . \cup\left\{A^{\omega} \rightarrow A^{\omega}\right\} \cup\left\{A^{\omega} \vee A^{\omega}\right\} \cup\left\{A^{\omega} \vee\left(F \backslash A^{\omega}\right)\right\} \cup\left\{\left(F \backslash A^{\omega}\right) \vee A^{\omega}\right\} .
\end{aligned}
$$

Observation 12 Let $\left(c_{n}\right),\left(d_{n}\right)$ and $\left(e_{n}\right)$ be three sequences of natural numbers, such that $c_{n} \leq d_{n}$ for all $n \in \mathbb{N}$. Suppose two new sequences are defined recursively as follows:

$$
x_{n}=c_{n}+\sum_{i=1}^{n-1} e_{i} \cdot x_{n-i}, \quad y_{n}=d_{n}+\sum_{i=1}^{n-1} e_{i} \cdot y_{n-i}
$$


Then $x_{n} \leq y_{n}$ for any $n \in \mathbb{N}$.

We will consider subset of $A^{\omega}$. We just omit the infinite part in the formula (15), which is written as '.... The omitted sets consists of formulas which are implications. The new set is denoted as $B^{5}$ and the superscript 5 is due to the fact that all intuitionistically valid implications, with the predecessor in $A^{n}$, for $n>5$, do not appear in $B^{5}$.

$$
\begin{aligned}
& B^{5}=\left\{A^{0} \rightarrow F\right\} \cup\left\{A^{1} \rightarrow\left(F \backslash\left(A^{0} \cup A^{2}\right)\right)\right\} \cup \ldots \\
& \cup\left\{A^{5} \rightarrow\left(F \backslash\left(A^{0} \cup A^{1} \cup A^{2} \cup A^{3} \cup A^{4} \cup A^{6}\right)\right)\right\} \cup \\
& \cup\left\{B^{5} \rightarrow B^{5}\right\} \cup\left\{B^{5} \vee B^{5}\right\} \cup\left\{B^{5} \vee\left(F \backslash B^{5}\right)\right\} \cup\left\{\left(F \backslash B^{5}\right) \vee B^{5}\right\}
\end{aligned}
$$

On the basis of Observation 12 we conclude that $\left|B_{n}^{5}\right| \leq\left|A_{n}^{\omega}\right|$ for any $n \in \mathbb{N}$. The generating function $g_{5}$, for $\left|B_{n}^{5}\right|$ is the following:

$$
\begin{aligned}
g_{5}= & {\left[f_{0} \cdot f+f_{1} \cdot\left(f-f_{0}-f_{2}\right)+f_{2} \cdot\left(f-f_{0}-f_{1}-f_{4}\right)+\right.} \\
& +f_{3} \cdot\left(f-f_{0}-f_{1}-f_{2}-f_{4}\right)+f_{4} \cdot\left(f-f_{0}-f_{1}-f_{2}-f_{3}-f_{6}\right)+ \\
& \left.+f_{5} \cdot\left(f-f_{0}-f_{1}-f_{2}-f_{3}-f_{4}-f_{6}\right)\right] /(1-2 f) .
\end{aligned}
$$

The function $g_{5}$ is obtained from the generating functions for classes of formulas from $A L_{8}$. So, $z_{0}=1 / 16$ is its dominant singularity and its density exists.

Lemma 13 The density of the class $B^{5}$ exists and $\mu\left(B^{5}\right) \approx 0.7068$.

From Lemma 13 and Observations 11 and 12 we obtain a quite tight estimation of the density of truth of intuitionistic tautologies.

Theorem 14 If the density of the class $A^{\omega}$ exists, then it is estimated as follows:

$$
0.7068 \leq \mu\left(A^{\omega}\right) \leq 0.709011
$$

\section{Existence of the density}

In this section we prove that the density of truth $\mu\left(A^{\omega}\right)$ exists. We define two sequences of sets (and numbers) approximating the set $A^{\omega}$ (and $\left|A_{n}^{\omega}\right|$ ). Let us consider the sequence $\left(B_{n}^{2 k}\right)$ of smaller than $A_{n}^{\omega}$ sets defined analogously as the set $B_{n}^{5}$ in the previous section:

$$
\begin{aligned}
& B^{2 k}=\left\{A^{0} \rightarrow F\right\} \cup\left\{A^{1} \rightarrow\left(F \backslash\left(A^{0} \cup A^{2}\right)\right)\right\} \cup\left\{A^{2} \rightarrow\left(F \backslash\left(A^{0} \cup A^{1} \cup A^{4}\right)\right)\right\} \cup \ldots \\
& \ldots \cup\left\{A^{2 k} \rightarrow\left(F \backslash\left(A^{0} \cup A^{1} \cup \ldots \cup A^{2 k-2} \cup A^{2 k-1} \cup A^{2 k+2}\right)\right)\right\} \cup \\
& \left\{B^{2 k} \rightarrow B^{2 k}\right\} \cup\left\{B^{2 k} \vee B^{2 k}\right\} \cup\left\{B^{2 k} \vee\left(F \backslash B^{2 k}\right)\right\} \cup\left\{\left(F \backslash B^{2 k}\right) \vee B^{2 k}\right\} .
\end{aligned}
$$

The formula 19 defines the cardinalities of subsets of some set $B^{2 k}$, with $k \geq 2$, which is a proper subset of $A^{\omega}$. Hence on the basis of Observation 12 and inclusions 8 we get the following inclusions:

$$
B^{4} \subset B^{6} \subset \ldots \subset A^{\omega} \subset \ldots \subset\left[A^{5}\right) \subset\left[A^{3}\right) .
$$

Let us consider the sequence of compartments $\left[\mu\left(B^{2 k}\right), \mu\left(\left[A^{2 k-1}\right)\right)\right]$, for $k \geq 2$, and show that their 'lengths' tend to 0 . 


\section{Lemma 15}

$$
\lim _{k \rightarrow \infty}\left(\mu\left(\left[A^{2 k-1}\right)\right)-\mu\left(B^{2 k}\right)\right)=0
$$

Proof. We consider the numbers $\left|\left[A^{2 k-1}\right)_{n}\right|-\left|B_{n}^{2 k}\right|$. From 14 and 19 we obtain:

$$
\begin{aligned}
\left|\left[A^{2 k-1}\right)_{n}\right|-\left|B_{n}^{2 k}\right|= & 2 \sum_{i=1}^{n-1}\left|A_{i}^{2 k-2}\right| \cdot\left(\left|A_{n-i}^{2 k-4}\right|+\left|A_{n-i}^{2 k-3}\right|+\left|A_{n-i}^{2 k}\right|\right)+ \\
& +\sum_{i=1}^{n-1}\left|A_{i}^{2 k}\right| \cdot\left(\left|A_{n-i}^{2 k-3}\right|+\left|A_{n-i}^{2 k-1}\right|+\left|A_{n-i}^{2 k+2}\right|\right)+ \\
& +2 \sum_{i=1}^{n-1}\left|F_{i}\right| \cdot\left(\left|\left[A^{2 k-1}\right)_{n-i}\right|-\left|B_{n-i}^{2 k}\right|\right)-\sum_{i=1}^{n-1}\left|A_{i}^{2 k-1}\right| \cdot\left|\left[A^{2 k-1}\right)_{n-i}\right|
\end{aligned}
$$

The numbers $\sum_{i=1}^{n-1}\left|A_{i}^{2 k-1}\right| \cdot\left|\left[A^{2 k-1}\right)_{n-i}\right|$ are non-negative, so on the base of Observation 12 we may consider larger numbers $\left|C_{n}^{k}\right|$ :

$$
\begin{aligned}
\left|C_{n}^{k}\right|= & 2 \sum_{i=1}^{n-1}\left|A_{i}^{2 k-2}\right| \cdot\left(\left|A_{n-i}^{2 k-4}\right|+\left|A_{n-i}^{2 k-3}\right|+\left|A_{n-i}^{2 k}\right|\right)+ \\
& +\sum_{i=1}^{n-1}\left|A_{i}^{2 k}\right| \cdot\left(\left|A_{n-i}^{2 k-3}\right|+\left|A_{n-i}^{2 k-1}\right|+\left|A_{n-i}^{2 k+2}\right|\right)+2 \sum_{i=1}^{n-1}\left|F_{i}\right| \cdot\left|C_{n-i}^{k}\right| .
\end{aligned}
$$

The numbers $\left|C_{n}^{k}\right|$ characterize the set $C^{k}$ consisting of formulas being disjunctions between formulas from $A^{2 k-2}$ and $A^{2 k-4} \cup A^{2 k-3} \cup A^{2 k}$, and implications from $A^{2 k}$ to $A^{2 k-3} \cup A^{2 k-1} \cup A^{2 k+2}$, and disjunctions between formulas from $C^{k}$ and formulas from $F$.

From the above we obtain formulas defining the generating functions $f_{C^{k}}$ for the numbers $\left|C_{n}^{k}\right|$ :

$$
f_{C^{k}}=\left[f_{2 k} \cdot\left(f_{2 k-3}+f_{2 k-1}+f_{2 k+2}\right)+2 f_{2 k-2} \cdot\left(f_{2 k-4}+f_{2 k-3}+f_{2 k}\right)\right] /(1-2 f) .
$$

The function $f_{C^{k}}$ is defined by functions with dominant singularity at $z_{0}=1 / 16$ (see proof of Theorem 6. So, it has the same dominant singularity. The density of the class $C^{k}$ can be computed as follows:

$$
\mu\left(C^{k}\right)=\frac{f_{C^{k}}^{\prime}\left(\frac{1}{16}\right)}{f^{\prime}\left(\frac{1}{16}\right)} .
$$

We show that the values of $f_{C^{k}}^{\prime}\left(\frac{1}{16}\right)$ tend to 0 when $k$ tends to infinity. For simplicity, we introduce a new symbol $h_{k}:=f_{2 k} \cdot\left(f_{2 k-3}+f_{2 k-1}+f_{2 k+2}\right)+2 f_{2 k-2} \cdot\left(f_{2 k-4}+f_{2 k-3}+f_{2 k}\right)$. Then, from [25, we have:

$$
f_{C^{k}}^{\prime}\left(\frac{1}{16}\right)=\frac{h_{k}^{\prime}\left(\frac{1}{16}\right) \cdot\left(1-2 f\left(\frac{1}{16}\right)\right)-h_{k}\left(\frac{1}{16}\right) \cdot\left(-2 f^{\prime}\left(\frac{1}{16}\right)\right)}{\left(1-2 f\left(\frac{1}{16}\right)\right)^{2}} .
$$


The values of $f\left(\frac{1}{16}\right)$ and $f^{\prime}\left(\frac{1}{16}\right)$ exist and are constant. To prove that

$$
\lim _{k \rightarrow \infty} h_{k}^{\prime}\left(\frac{1}{16}\right)=0 \quad \text { and } \quad \lim _{k \rightarrow \infty} h_{k}\left(\frac{1}{16}\right)=0 .
$$

we prove that

$$
\lim _{k \rightarrow \infty} f_{k}^{\prime}\left(\frac{1}{16}\right)=0 \text { and } \quad \lim _{k \rightarrow \infty} f_{k}\left(\frac{1}{16}\right)=0 .
$$

It is straightforward to observe that 28 yields $\sqrt{27}$. From Theorem 6 it follows that $\mu\left(A^{k}\right)$ exists for each $k \in \mathbb{N}$. By $\sqrt{7}$, the series $\sum_{k=0}^{\infty} \mu\left(A^{k}\right)$ is convergent and hence $\lim _{k \rightarrow \infty} \mu\left(A^{k}\right)=0$. So, from the transfer lemma (we know that the functions $f_{k}$ have the same dominant singularity) we obtain:

$$
\lim _{k \rightarrow \infty} f_{k}^{\prime}\left(\frac{1}{16}\right)=0 \text {. }
$$

Similarly, let us consider the series $\sum_{k=0}^{\infty} f_{k}\left(\frac{1}{16}\right)$. This series is bounded by $f\left(\frac{1}{16}\right)=\frac{1}{2}$ and the values $f_{k}\left(\frac{1}{16}\right)$ are non-negative ${ }^{(i)}$ so it also must be convergent. Hence:

$$
\lim _{k \rightarrow \infty} f_{k}\left(\frac{1}{16}\right)=0
$$

By Theorem 14 and Lemma 15 we get:

Theorem 16 The density of the class $A^{\omega}$ exists and is about $70 \%$.

Let us compare the above result with the one we can get for the classical logic:

Observation 17 The algebra $A L_{4}$ is the Lindenbaum algebra of the classical logic $\{\rightarrow, \vee, \perp\}$ in one variable. Hence

$$
\mu\left(C L_{\{p\}}^{\{\rightarrow, \vee, \perp\}}\right)=\mu\left(\left[A^{3}\right)\right) \approx 0.736
$$

By Theorem 14 and Observation 17 we immediately have:

Theorem 18 The relative density of intuitionistic tautologies among classical ones in the language $\{\rightarrow, \vee, \perp, p\}$ is more than $96 \%$.

We conjecture that the density of truth for $I N T_{m}^{\{\rightarrow, \vee, \perp\}}$ exists for any $m \geq 1$, where $m$ is the number of variables occurring in the language. The following problem appears:

Problem 1 Whether, or not, the logics $I N T_{m}^{\{\rightarrow, \vee, \perp\}}$ and $C L_{m}^{\{\rightarrow, \vee, \perp\}}$ are asymptotically identical.

It seems to be important to work out a new and effective method for calculation (or estimation) the density of truth in the case of logics with infinite number of non-equivalent formulas. Any semantical approach to this question usually involves extremely complicated calculations. More promising seem to be syntactical approaches where one can analyze shapes of binary trees. However, these methods also need some refinement as, for instance, formulas from $\{\rightarrow, \vee\}$ have not been yet investigated as binary trees.

(i) It could be justified as follows: for each $i \in \mathbb{N}, f_{i}(1 / 16) \geq 0$ because $f_{i}(z)=\sum_{n=0}^{\infty} a_{i n} z^{n}$ and the series is convergent at $z_{0}=\frac{1}{16}$ and then the $\operatorname{sum} \sum_{n=0}^{\infty} a_{i n}\left(\frac{1}{16}\right)^{n}$ is also non-negative. 


\section{References}

[1] B. Chauvin B., Flajolet P., Gardy D., Gittenberger B. And/Or trees revisited, Combinatorics, Probability and Computing, 13(4-5), July-September 2004, pp 475-497.

[2] Flajolet P. and Sedgewick R. Analitic combinatorics: functional equations, rational and algebraic functions, INRIA, Number 4103, 2001.

[3] Flajolet P. and Odlyzko A. M. Singularity analysis of generating functions, SIAM J. on Discrete Math., 3(2), 1990, pp 216-240.

[4] Fournier H., Gardy D., Genitrini A., Zaionc M. Classical and intuitionistic logic are asymptotically identical,Lecture Notes in Computer Science 4646, pp. 177-193.

[5] Gardy D. and Woods A.R. And/or tree probabilities of Boolean functions, Discrete Mathematics and Theoretical Computer Science, 2005, pp 139-146.

[6] Kostrzycka Z. On the density of implicational parts of intuitionistic and classical logics, Journal of Applied Non-Classical Logics, Vol. 13, Number 3, 2003, pp 295-325.

[7] Kostrzycka Z. and Zaionc M. Statistics of intuitionistic versus classical logics, Studia Logica, Vol. 76, Number 3, 2004, pp 307-328.

[8] Kostrzycka Z. and Zaionc M. Asymptotic densities in logic and type theory, Studia Logica, Vol. 88, 2008, pp 385-403.

[9] Lefmann H., Savický P. Some typical properties of large and/or boolean formulas, Random Structures and Algorithms, 10, 1997, pp 337-351.

[10] Mints G. A Short Introduction to Intuitionistic Logic. The University Series in Mathematics, Kluwer Academic/Plenum Publishers, Stanford 2000.

[11] Moczurad M., Tyszkiewicz J., Zaionc M. Statistical properties of simple types, Mathematical Structures in Computer Science, vol 10, 2000, pp 575-594.

[12] Wilf H.S. Generatingfunctionology. Second edition. Academic Press, Boston 1994.

[13] Woods A.R. Coloring Rules for Finite Trees, and Probabilities of Monadic Second Order Sentences, Random Structures Algorithms 10, (4), 1997, 453-485. 